\title{
Differential diffusion effects on density-driven instability of reactive flows in porous media
}

\author{
Timan Lei and Kai H. Luo ${ }^{*}$ \\ Department of Mechanical Engineering, University College London, Torrington Place, \\ London WC1E 7JE, United Kingdom
}

(Received 15 November 2019; accepted 5 March 2020; published 31 March 2020)

\begin{abstract}
Based on the lattice Boltzmann (LB) method, pore-scale simulations are performed to investigate the differential diffusion effects on the density-driven instability (DI) with chemical reaction $A+B \rightarrow C$ in porous media. A partially miscible stratification is considered, and thus only solutes from the top fluid can diffuse down into the host fluid in pore spaces. Tests with different values of the Rayleigh number $\mathrm{Ra}_{r}$ and the diffusion coefficient $D_{r}$ of species $r(r=A, B, C)$ are considered. The results demonstrate eight distinct scenarios of DI, and four of them are not observed in equal diffusivity simulations. Two differential diffusion effects, namely, the double-diffusive (DD) and the diffusive-layer convection (DLC) mechanisms, can act upon the gravity field and give rise to new fingering phenomena. The DD mechanism comes into play and results in a local minimum density layer if $\mathrm{Ra}_{B} / \mathrm{Ra}_{C}$ is small and $D_{B}>D_{C}$; and DLC becomes significant and brings in a local maximum density layer if $\mathrm{Ra}_{B} / \mathrm{Ra}_{C}$ is large and $D_{B}<D_{C}$. On one hand, when fluid density increases with dissolved $A$, the DD-induced minimum can act as an inhibiting barrier to suppress fingering propagation, although it can be eventually penetrated by fingering tips; and the DLC-induced maximum can introduce the second DI below the first one. On the other hand, when the dissolution of $A$ contributes to decreasing fluid density, both the DD-induced minimum and the DLC-induced maximum can help trigger the development of DI. Finally, quantitative results are provided to indicate that fingering propagates into the host fluid more deeply with larger $D_{B} / D_{C}$, and the dissolution of $A$ decreases with the increasing difference between $D_{B}$ and $D_{C}$.
\end{abstract}

DOI: 10.1103/PhysRevFluids.5.033903

\section{INTRODUCTION}

A partially miscible stratification is typically composed of two miscible solutions (labeled as 1 and 2), and a solute $A$ from the top fluid 1 diffuses into the fluid 2 below, but no mass transfers in the reverse direction. In a porous medium, such a stratification develops when the top boundary is partially miscible, and species $A$ from the above fluid 1 can diffuse down into the host fluid 2, filling the pore spaces. Dissolved $A$ can react with another solute $B$ in the host fluid following the $A+B \rightarrow C$ scheme, and all three chemical species $A, B$, and $C$ usually diffuse at different rates. The coupling effects of diffusion and reaction can moderate species concentrations and thus distributions of viscosity [1,2], salinity [3], and density [4] in the host fluid. Subsequently, different types of density-driven instability (DI) dynamics may be introduced by unstable density distributions; even no injection velocity is involved in this partially miscible stratification [4]. Such an instability accompanied by convection can drive efficient mass and heat transport, leading to the improved storage efficiency and security of species $A$ in the host fluid. It is thus at the heart of carbon dioxide

\footnotetext{
*Corresponding author: k.luo@ucl.ac.uk
} 
$\left(\mathrm{CO}_{2}\right)$ sequestration and has also gained considerable interest in groundwater management $[5,6]$ and enhanced oil recovery [7]. In this regard, fundamental understanding of how reaction and differential diffusion affect DI dynamics in a porous medium with a partially miscible top boundary is crucial to helping predict and design relevant applications.

During the past several years, investigations have been carried out to explore the effects of reaction $A+B \rightarrow C$ on the development of DI in a porous medium with a partially miscible top boundary. For instance, some experiments have been performed to observe the characteristics of DI in ester-alkaline and $\mathrm{CO}_{2}$-alkaline solutions separately [8-11]. Captured experimental data have showed that different reactions can either inhibit or accelerate the development of DI with respect to the chemicals containing in the host fluid. Considering that the whole extent of DI may not be captured in the above experiments $[10,12]$, theoretical studies were performed simultaneously to analyze DI with reaction $A+B \rightarrow C$ [13-16]. Loodts et al. [16] theoretically provided a classification of four types of density profiles, with each one potentially representing a kind of DI dynamics. But these theoretical works focused on the early-stage development of DI and completely ignored the nonlinear fingering growth during the late period. More recently, numerical simulations have been conducted under the guidance of existing theoretical predictions. Budroni et al. [11] numerically modeled both the stabilized and destabilized fingering phenomena with reaction, and their results qualitatively verified both experimental observations and theoretical predictions. Simulation results of Loodts et al. [4] provided the spatial-temporal properties of four types of DI with reaction $A+B \rightarrow C$, which matched well with their theoretical predictions.

Seen from the above experimental, theoretical, and numerical results, chemical reaction can modify fluid density, and subsequently stabilize, destabilize, or even trigger the development of DI in porous media. These findings are useful, but they are all based on the assumption that the three species diffuse equally. It has been reported that differential diffusion effects need to be taken into account to interpret experimental results $[17,18]$. Besides, in the context of $\mathrm{CO}_{2}$ sequestration, solutes in the host fluid are likely to have different diffusion coefficients [19]. Thus, it is necessary to consider a more general case with the three chemical species diffusing at different rates. Until now, two types of DI introduced by differential diffusion have been reported under nonreactive conditions [20,21]. Explicitly, if a less dense solution of a slow diffusing species overlies a denser one of a fast-diffusing solute, an accumulation zone can be created in the top layer with a depletion area forming below, which is known as a double-diffusive (DD) mechanism. These two areas feature a locally unstable stratification and subsequently trigger the development of DI. In parallel, the diffusive-layer convection (DLC) mechanism occurs when the upper component diffuses faster than the lower one, and a depletion zone followed by an accumulation one can be built up, leading to the appearance of DI. Indeed, research has also been conducted to investigate the effects of these two differential diffusion mechanisms on the development of DI in porous media, with reaction $A+B \rightarrow C$ being considered. For example, Trevelyan et al. [22] theoretically predicted that, for a miscible interface between two reactive solutions, the inclusion of differential diffusion could give up to 62 types of density profiles. Similarly, by varying the Rayleigh numbers and diffusion rates of three chemical species, Loodts et al. [23] theoretically identified eight different density profiles for a partially miscible stratification in a porous media. After that, they assumed that dissolved A can only increase the density of the host fluid and numerically verified four types of theoretical density profiles [19].

In summary, existing results have improved our understanding of the effects of differential diffusion on DI with the reaction $A+B \rightarrow C$, but two limitations should be noted. First, a comprehensive numerical study on such a problem is needed. Although theoretical analyses by Loodts et al. [23] help to predict potential fingering scenarios, numerical simulations are necessary to illustrate the nonlinear fingering properties during the late stage. In addition, existing simulations in Ref. [19] are performed under the assumption that fluid density increases with the dissolution of $A$, and a large part of the parameter space remains unexplored. Second, pore-scale simulations are missing. Owing to the complex geometries of porous media, previous numerical studies were conducted on a representative elementary volume scale where a number of assumptions are required 


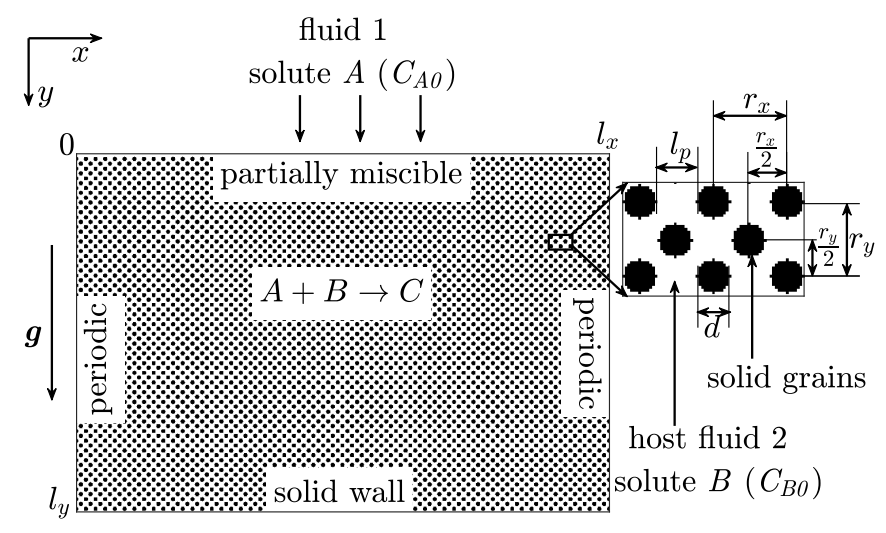

FIG. 1. The computational configuration.

[24]. During the past three decades, the lattice Boltzmann (LB) method has become an attractive alternative to conventional solvers for studying various fluid flow problems at pore scale $[25,26]$. This is due to its simple implementation, high parallelism, and ability to handle complex boundary conditions. Actually, in our recent work [27], an LB model has been developed to successfully reproduce four types of DI dynamics with the reaction $A+B \rightarrow C$ at pore scale, but different diffusion rates of the three species are not involved. Therefore, to fill these gaps, the LB method is further applied to simulate the development of DI coupled with reaction $A+B \rightarrow C$ in a porous medium with a partially miscible top boundary at pore scale, and the effects of differential diffusion are mainly discussed.

\section{MATHEMATICAL MODEL}

In this study, DI between two reactive solutions (labeled 1 and 2) is investigated in a twodimensional (2D) homogeneous porous medium with porosity $\phi=0.69$. As displayed in Fig. 1 , the computational domain is $0 \leqslant x \leqslant l_{x}=1$ and $0 \leqslant y \leqslant l_{y}=2 / 3$, and the porous network contains a staggered array of circular grains with a uniform diameter $d$. Every grain center obeys a regular staggered distribution, and the closest center-to-center distances between two cylinders in $x$ and $y$ directions are $r_{x}$ and $r_{y}$, respectively. The single pore size can be then calculated as $l_{p}=r_{x}-d$. In this medium, fluid 1 of species $A$ is initially placed in contact with the host fluid 2 in pore spaces along a horizontal interface at $y=0$, with $y$ pointing along the gravity field. These two solutions are considered miscible and incompressible, and the host fluid contains a dissolved reactant $B$ in initial concentration $C_{B 0}$. During the course of time, $A$ diffuses into the host fluid 2 and reacts with $B$ to yield a product $C$ following the $A+B \rightarrow C$ scheme. The reaction rate $R$ is calculated as [4]

$$
R=k C_{A} C_{B},
$$

with $k$ being the kinetic reaction constant and $C_{r}$ being the concentration of species $r$. All three chemical species can contribute to changes in fluid density $\rho$. With the well-known Boussinesq approximation, $\rho$ is considered to be constant $\rho_{0}=1$ except in the body force term, where it is taken as [4]

$$
\rho=\rho_{0}+\rho_{0}\left(\beta_{A} C_{A}+\beta_{B} C_{B}+\beta_{C} C_{C}\right),
$$

and $\beta_{r}$ is the concentration expansion coefficient of species $r$.

In this work, the top boundary $(y=0)$ is assumed to be partially miscible [4]. Solute $A$ can dissolve from fluid 1 into the host fluid 2, but no mass transfer takes place in the reverse direction. Thus, this work focuses on the fluid motion and concentration evolutions in the host fluid, which 
can be described by the incompressible Navier-Stokes (NS) and the convection-diffusion-reaction (CDR) equations:

$$
\begin{gathered}
\nabla \cdot \boldsymbol{u}=0 \\
\rho_{0}\left(\frac{\partial \boldsymbol{u}}{\partial t}+\boldsymbol{u} \cdot \nabla \boldsymbol{u}\right)=-\nabla p+\nabla \cdot\left(v \rho_{0} \nabla \boldsymbol{u}\right)+\boldsymbol{F} \\
\frac{\partial C_{A}}{\partial t}+\boldsymbol{u} \cdot \nabla C_{A}=D_{A} \nabla^{2} C_{A}-R \\
\frac{\partial C_{B}}{\partial t}+\boldsymbol{u} \cdot \nabla C_{B}=D_{B} \nabla^{2} C_{B}-R \\
\frac{\partial C_{C}}{\partial t}+\boldsymbol{u} \cdot \nabla C_{C}=D_{C} \nabla^{2} C_{C}+R
\end{gathered}
$$

where $\boldsymbol{u}=(u, v)$ and $p$ are the fluid velocity and pressure, respectively, $t$ is the time, and $v$ is the kinematic viscosity. With the constant force term $\rho_{0} \boldsymbol{g}$ being absorbed into the pressure term $\nabla p$ [28], the buoyancy force is expressed as

$$
\boldsymbol{F}=\rho_{0} \boldsymbol{g}\left(\beta_{A} C_{A}+\beta_{B} C_{B}+\beta_{C} C_{C}\right)
$$

where $g$ is the acceleration vector of gravity. To solve the present governing equations (3)-(7), initial and boundary conditions are given as

$$
\begin{aligned}
C_{A}(x, 0 \leqslant y \leqslant \vartheta, 0) & =C_{A 0}=1, \quad C_{A}(x, y>\vartheta, 0)=0, \\
C_{B}(x, y, 0) & =C_{B 0}, \quad C_{C}(x, y, 0)=0
\end{aligned}
$$

and

$$
\begin{aligned}
C_{r}(0, y, t) & =C_{r}\left(l_{x}, y, t\right), \quad \boldsymbol{u}(0, y, t)=\boldsymbol{u}\left(l_{x}, y, t\right), \\
\nabla C_{r}\left(x, l_{y}, t\right) & =(0,0), \quad \boldsymbol{u}\left(x, l_{y}, t\right)=(0,0), \\
C_{A}(x, 0, t) & =C_{A 0}, \quad \nabla C_{B, C}(x, 0, t)=(0,0), \quad \boldsymbol{u}(x, 0, t)=(0,0), \\
\nabla C_{r}\left(x_{s}, y_{s}, t\right) & =(0,0), \quad \boldsymbol{u}\left(x_{s}, y_{s}, t\right)=(0,0),
\end{aligned}
$$

with $\boldsymbol{x}=\left(x_{s}, y_{s}\right)$ representing the solid matrix interface. Note that following the local chemical equilibrium assumption, $C_{A}$ at the top boundary $(y=0)$ is considered to be constant and equal to the solubility of species $A$ in the host fluid [4]. In addition, a small perturbation $\vartheta$ is added to the initial distribution of $A$, and the amplitude of this parameter randomly varies along the $x$ direction within the range $\left[0,0.0067 l_{x}\right]$.

Equations (3)-(7) can be expressed in a dimensionless form by introducing the characteristic length $L$, velocity $U$, time $T$, and concentration $C_{d}$ as

$$
L=l_{x}, \quad U=\sqrt{g \beta_{A} L C_{A 0}}, \quad T=L / U, \quad C_{d}=C_{A 0} .
$$

In terms of the following nondimensional variables,

$$
\begin{aligned}
& \boldsymbol{u}^{*}=\frac{\boldsymbol{u}}{U}, \quad p^{*}=\frac{p}{\rho_{0} U^{2}}, \quad C_{r}^{*}=\frac{C_{r}}{C_{A 0}}, \quad \eta=\frac{C_{B 0}}{C_{A 0}}, \quad D_{B}^{*}=\frac{D_{B}}{D_{A}}, \quad D_{C}^{*}=\frac{D_{C}}{D_{A}}, \\
& x^{*}=\frac{x}{L}, \quad y^{*}=\frac{y}{L}, \quad t^{*}=\frac{t}{T}, \quad \rho^{*}=\frac{\rho-\rho_{0}}{\rho_{0} \beta_{A} C_{A 0}}, \quad \boldsymbol{F}^{*}=\frac{\boldsymbol{F}}{\rho_{0} U^{2} / L}, \\
& R^{*}=\mathrm{DaC}_{A}^{*} C_{B}^{*}, \quad \mathrm{Ra}_{r}=\frac{g \beta_{r} C_{A 0} L^{3}}{v D_{A}}, \quad \mathrm{Sc}=\frac{v}{D_{A}}, \quad \mathrm{Da}=\frac{k C_{A 0} L}{U},
\end{aligned}
$$


where the asterisked variables are the corresponding dimensionless ones, the dimensionless equations (3)-(7) read

$$
\begin{gathered}
\nabla \cdot \boldsymbol{u}^{*}=0, \\
\frac{\partial \boldsymbol{u}^{*}}{\partial t^{*}}+\boldsymbol{u}^{*} \cdot \nabla \boldsymbol{u}^{*}=-\nabla p^{*}+\sqrt{\frac{\mathrm{Sc}}{\mathrm{Ra}_{A}} \nabla \cdot\left(\nu \nabla \boldsymbol{u}^{*}\right)+\boldsymbol{F}^{*},} \\
\frac{\partial C_{A}^{*}}{\partial t^{*}}+\boldsymbol{u}^{*} \cdot \nabla C_{A}^{*}=\frac{1}{\sqrt{\mathrm{Ra}_{A} \mathrm{Sc}}} \nabla^{2} C_{A}^{*}-R^{*}, \\
\frac{\partial C_{B}^{*}}{\partial t^{*}}+\boldsymbol{u}^{*} \cdot \nabla C_{B}^{*}=\frac{D_{B}^{*}}{\sqrt{\mathrm{Ra}_{A} \mathrm{Sc}}} \nabla^{2} C_{B}^{*}-R^{*} \\
\frac{\partial C_{C}^{*}}{\partial t^{*}}+\boldsymbol{u}^{*} \cdot \nabla C_{C}^{*}=\frac{D_{C}^{*}}{\sqrt{\mathrm{Ra}_{A} \mathrm{Sc}}} \nabla^{2} C_{C}^{*}+R^{*}
\end{gathered}
$$

As can be seen, the behaviors of DI with reaction $A+B \rightarrow C$ and differential diffusion are related to the Rayleigh numbers $\mathrm{Ra}_{r}$, the diffusion coefficients $D_{r}^{*}$, the Schmidt number Sc, the Damköhler number $\mathrm{Da}$, and the initial concentration ratio $\eta$.

In this work, the above governing equations (3)-(7) are solved by a two-dimensional ninevelocity multiple-relaxation time (MRT) LB model, which can avoid the unphysical dependence of permeability on viscosity for pore-scale simulations [29-32]. An MRT LB model has been proposed to handle the development of DI with reaction $A+B \rightarrow C$ in our recent work [27], and it is further extended here for pore-scale simulations of DI coupled with both reaction and differential diffusion effects. More details about this model are introduced in Sec. III A. During LB simulations, the boundary conditions in Eq. (10) are treated as follows. The no-slip velocity and impermeable concentration conditions at the bottom solid wall and the porous matrix interface are realized by the halfway bounce-back scheme [33,34], and the partially miscible condition at the top boundary is treated with the nonequilibrium extrapolation scheme [24].

\section{RESULTS AND DISCUSSION}

In this section, the development of DI with reaction $A+B \rightarrow C$ and differential diffusion is simulated in a homogeneous medium based on the MRT LB method. As theoretically predicted in Ref. [23], such a problem can be characterized by two numbers: the buoyancy ratio $\mathrm{Ra}_{B} / \mathrm{Ra}_{C}$ quantifying the relative contributions of $B$ and $C$ to fluid density, and the diffusion coefficient ratio $D_{B} / D_{C}$ of these two species. Thus, in the following simulations, the Schmidt number, Damköhler number, and initial concentration ratio are fixed as $\mathrm{Sc}=100, \mathrm{Da}=5$, and $\eta=1$, respectively, and different values of $\mathrm{Ra}_{r}$ and $D_{r}$ are selected to change test conditions. In addition, grid convergence tests are carried out, and a mesh of size $N_{x} \times N_{y}=1500 \times 1000$ is chosen to cover the computational domain in Fig. 1. Other geometrical parameters are set as $d=12 \delta_{x}, r_{x}=r_{y}=27 \delta_{x}$, and $l_{p}=15 \delta_{x}$, respectively, with the lattice spacing being $\delta_{x}=l_{x} / N_{x}$.

\section{A. General phenomena}

The general phenomena of DI coupled with impact of chemistry and differential diffusion are first investigated. To gain a comprehensive understanding, two Rayleigh numbers of species $A\left(\operatorname{Ra}_{A}= \pm 10^{9}\right)$ are considered, and thus fluid density can increase $\left(\operatorname{Ra}_{A}=10^{9}\right)$ or decrease $\left(\mathrm{Ra}_{A}=-10^{9}\right)$ with the dissolution of $A$. These two tests are both simulated at different values of diffusion ratio $D_{B} / D_{C}$, and a subsection of $\mathrm{Ra}_{B} / \mathrm{Ra}_{C}$ is modeled for each given $D_{B} / D_{C}$. After a series of simulations and comparisons, the development of DI can be delineated into four regimes for $\mathrm{Ra}_{A}>0$ as: $D_{B} / D_{C}<1$ with $\mathrm{Ra}_{B} / \mathrm{Ra}_{C}<\mathrm{Ra}_{B} / \mathrm{Ra}_{C_{S}}$ (case P1) or $\mathrm{Ra}_{B} / \mathrm{Ra}_{C}>\mathrm{Ra}_{B} / \mathrm{Ra}_{C_{S}}$ (case P2); $D_{B} / D_{C}>1$ with $\mathrm{Ra}_{B} / \mathrm{Ra}_{C}<\mathrm{Ra}_{B} / \mathrm{Ra}_{C_{S}}$ (case P3) or $\mathrm{Ra}_{B} / \mathrm{Ra}_{C}>\mathrm{Ra}_{B} / \mathrm{Ra}_{C}$ (case P4). Similarly, 
TABLE I. Parameters for cases P2-P3 and P6-P7.

\begin{tabular}{lccc}
\hline \hline Cases & \multicolumn{3}{c}{ Parameters } \\
\hline P2 & $\mathrm{Ra}_{A}=10^{9} ;$ & $D_{B} / D_{C}=0.1 ;$ & $\mathrm{Ra}_{B} / \mathrm{Ra}_{C}=1.0 ;$ \\
P3 & $\mathrm{Ra}_{A}=10^{9} ;$ & $D_{B} / D_{C}=5.0 ;$ & $\mathrm{Ra}_{B} / \mathrm{Ra}_{C}=1.0 ;$ \\
P6 & $\mathrm{Ra}_{A}=-10^{9} ;$ & $D_{B} / D_{C}=0.1 ;$ & $\mathrm{Ra}_{B} / \mathrm{Ra}_{C}=1.0 ;$ \\
P7 & $\mathrm{Ra}_{A}=-10^{9} ;$ & $D_{B} / D_{C}=5.0 ;$ & $\mathrm{Ra}_{B} / \mathrm{Ra}_{C}=1.0 ;$ \\
\hline
\end{tabular}

four cases P5-P6 can also be identified for $\mathrm{Ra}_{A}<0$, depending on the vales of $D_{B} / D_{C}$ and $\mathrm{Ra}_{B} / \mathrm{Ra}_{C}$. Note that $\mathrm{Ra}_{B} / \mathrm{Ra}_{C}$ is the switch threshold between two cases for a given $D_{B} / D_{C}$ and $\mathrm{Ra}_{A}$, and it increases with $D_{B} / D_{C}$. The present classification containing eight types of DI is generally similar to the theoretical prediction in Ref. [23]. It should be emphasized that the general fingering characteristics of cases P1, P4, P5, and P8 are consistent with those of cases R1-R4 in our recent equal diffusivity simulations in Ref. [27], and they are mainly determined by chemical reaction. Even though these four cases may be slightly influenced by differential diffusion, they are not introduced here for brevity, and more descriptions can be found in Ref. [27]. Therefore, the effects of differential diffusion on fingering phenomena are discussed for cases P2, P3, P6, and P7 as follows, and a few specific test conditions are provided in Table I.

CaseP2. Simulations are first performed for case P2, and the development of DI is clearly observed in each test. As an example, density evolutions at $D_{B} / D_{C}=0.1, \mathrm{Ra}_{A}=10^{9}$, and $\mathrm{Ra}_{B} / \mathrm{Ra}_{C}=1$ are shown in Fig. 2. The early diffusion can introduce a density stratification containing four layers: a denser fluid layer L1 near the top boundary overlies a local minimum density layer L2, and the following denser layer L3 is on top of a less dense one L4 [Fig. 2(a)]. Layers L2 and L3 are introduced by the difference between the diffusion rates of $B$ and $C$, which is referred to as a DLC mechanism [20]. To be specific, species $A$ reacts with $B$ to yield $C$ mainly at the reaction front $(\mathrm{RF})$, where the largest reaction rate takes place and two reactants are almost consumed. Then, $C$ diffuses downwards from RF without being fully replaced by $B\left(D_{B} / D_{C}<1\right)$, and a local species-depleted (SD) and local species-rich (SR) areas are subsequently generated at and below RF, respectively [Fig. 3(b)]. In this case with $\mathrm{Ra}_{B} / \mathrm{Ra}_{C}=1$, the contribution of species $C$ to fluid density is not large enough to change the density distribution, and thus a local minimum (L2) and a local maximum (L3) develop at the local SD and SR zones, respectively [Fig. 3(b)].

These initial fluid layers can form two buoyantly unstable stratifications: L1-L2 and L3-L4. After the short diffusion, the first DI develops from L1 near the top boundary [Fig. 2(b)] and corresponds to the stratification L1-L2. This DI then experiences three classical fingering development stages: fingering growth, merge, and new fingering growth, but without penetrating the minimum density layer L2 [Figs. 2(c)-2(e)]. During the late period, the second DI becomes visible from L3 [Fig. 2(d)], and it is induced by the stratification L3-L4. In contrast to the main fingers of the first DI, the secondary fingers tend to grow individually or divide into antenna-shaped forms [Fig. 2(e)], which can be explained by the fast-diffusing $C$ in L3 [21]. The concentration fields in Figs. 2(f)-2(h) corresponding to the density field in Fig. 2(e) display that species $C$ contributes to the second DI and the division of secondary fingers, while the first DI is caused by fingers of $A$. Two fingering areas are separated by L2, and the propagation of the first DI is slowed down by the locally stable stratification L2-L3. In addition, horizontally averaged density profiles are calculated and provided in Fig. 3(a). It is found that, in the early period, the density curve shows a nonmonotonic pattern with a local minimum followed by a local maximum, which is consistent with the initial density field in Fig. 2. During the late stage, the averaged density line transfers into a monotonically decreasing one, although the local minimum and maximum layers still exist in Fig. 2. The most likely explanation for this change is that the development of the first DI can bring in large nonlinearities to deform the local minimum and maximum layers. 


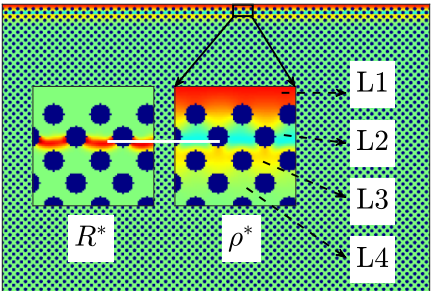

(a) $t^{*}=30$

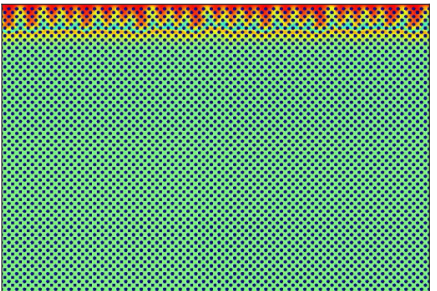

(b) $t^{*}=120$

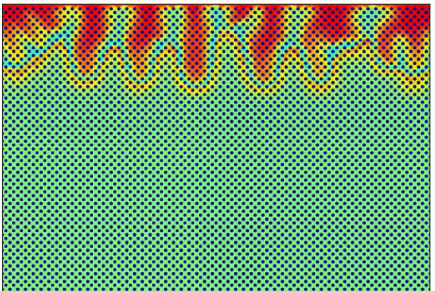

(c) $t^{*}=300$

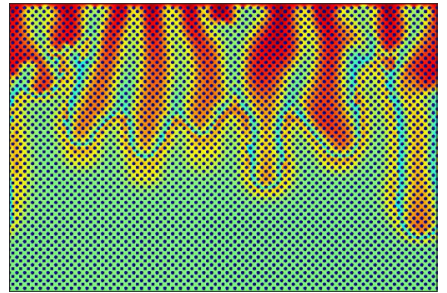

(d) $t^{*}=600$

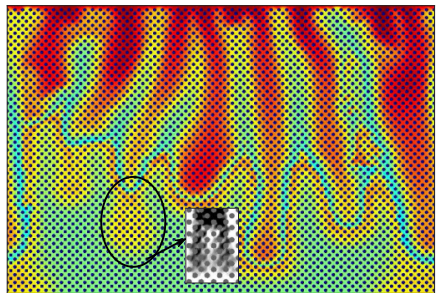

(e) $t^{*}=840$
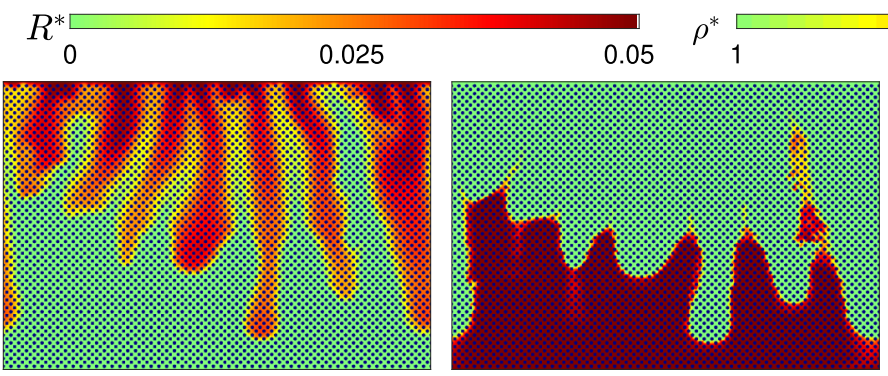

(f) $t^{*}=840 \quad C_{A}^{*}$

(g) $t^{*}=840 \quad C_{B}^{*}$
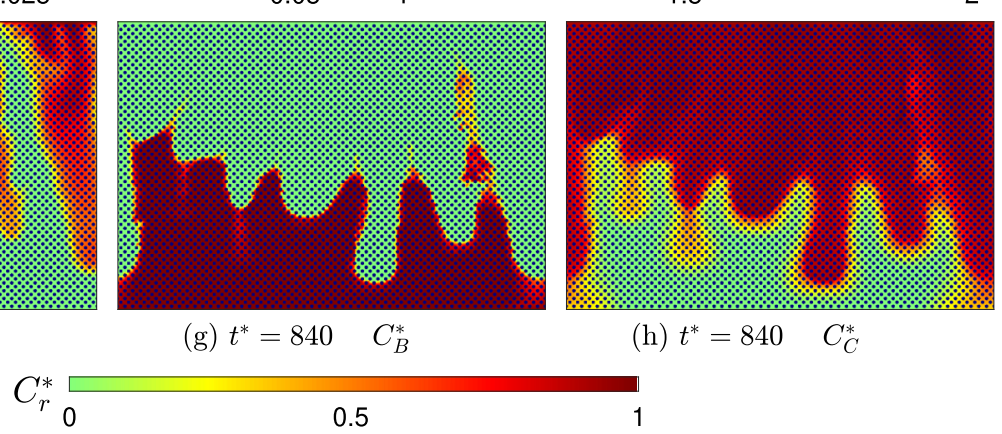

(h) $t^{*}=840 \quad C_{C}^{*}$

FIG. 2. Density (a)-(e) and concentration (f)-(h) distributions in homogeneous media for case P2 with $\mathrm{Ra}_{A}=10^{9}, D_{B} / D_{C}=0.1$, and $\mathrm{Ra}_{B} / \mathrm{Ra}_{C}=1.0$ at different time instants $t^{*}$. The density scale $\rho^{*}$ varies within the range 1-2; the concentrations $C_{r}^{*}$ scale between 0 and 1 ; and the reaction rate $R^{*}$ scales between 0 and 0.05 .
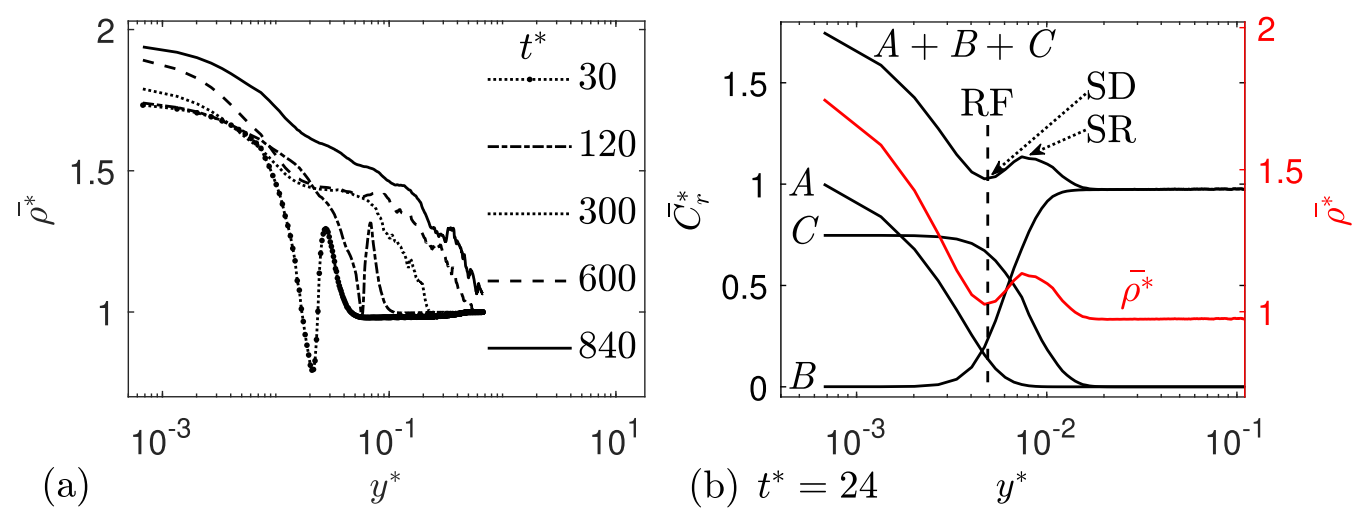

FIG. 3. Horizontally averaged density and species profiles in homogeneous media for case $\mathrm{P} 2$ with $\mathrm{Ra}_{A}=$ $10^{9}, D_{B} / D_{C}=0.1$, and $\mathrm{Ra}_{B} / \mathrm{Ra}_{C}=1.0$ at five different time instants (a) and time instant $t^{*}=24$ (b). 


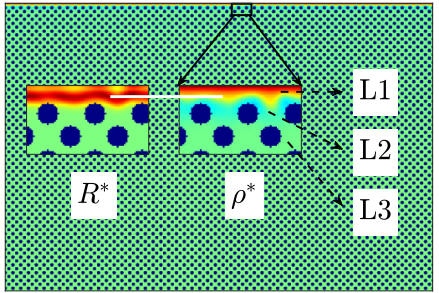

(a) $t^{*}=4$

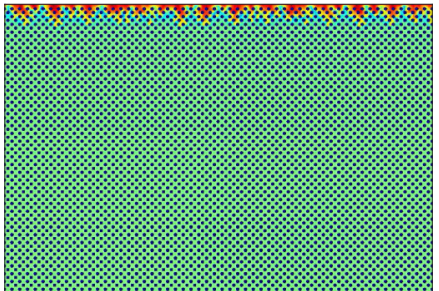

(b) $t^{*}=35$

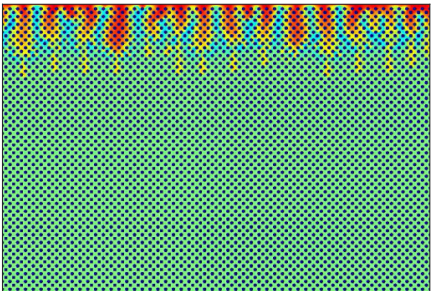

(c) $t^{*}=120$

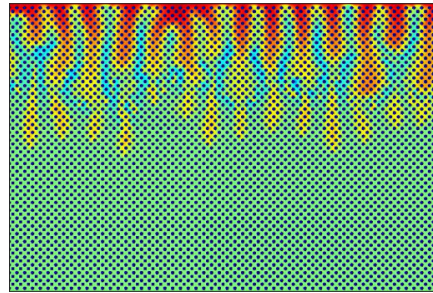

(d) $t^{*}=300$

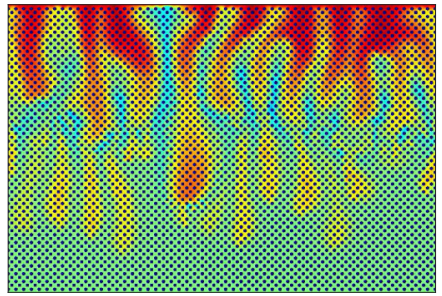

(e) $t^{*}=550$

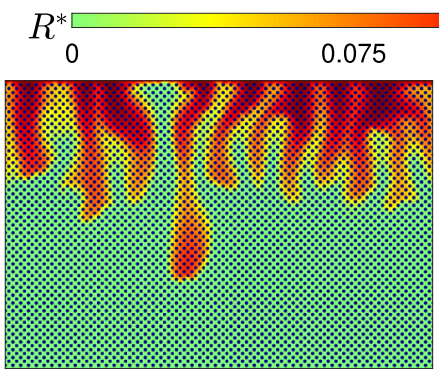

(f) $t^{*}=550 \quad C_{A}^{*}$

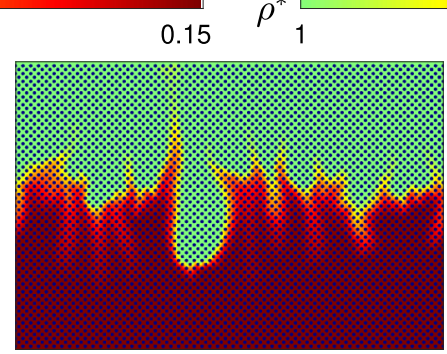

(g) $t^{*}=550 \quad C_{B}^{*}$

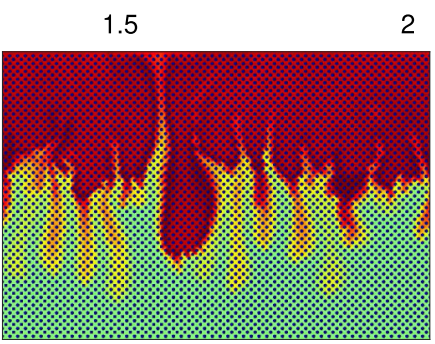

(h) $t^{*}=550 \quad C_{C}^{*}$

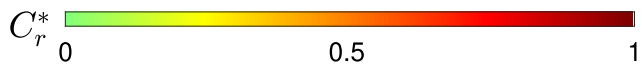

FIG. 4. Density (a)-(e) and concentration (f)-(h) distribution in homogeneous media for case P3 with $\mathrm{Ra}_{A}=10^{9}, D_{B} / D_{C}=5.0$, and $\mathrm{Ra}_{B} / \mathrm{Ra}_{C}=1.0$ at different time instants $t^{*}$. The density scale $\rho^{*}$ varies within the range 1-2; the concentrations $C_{r}^{*}$ scale between 0 and 1 ; and the reaction rate $R^{*}$ scales between 0 and 0.15 .

CaseP3. Fingering phenomena in case $\mathrm{P} 3$ are then simulated, and part of the results are provided in Fig. 4. A density stratification develops during the initial diffusion stage as a denser fluid layer L1 lies above a local minimum density layer L2 on top of a layer L3, and L2 locates below RF [Fig. 4(a)]. The formation of this initial stratification can be explained by another differential diffusion effect, namely, the DD mechanism [20]. Explicitly, the fast upward diffusion of $B$ and the slowly downward diffusion of $C$ contribute to the generation of a local SD area below RF [Fig. 5(b)]. Note that although the fast-diffusing $B$ can promote the accumulation of $C$ by the reaction $A+B \rightarrow C$ above $\mathrm{RF}$, no local SR region exists. This is because the dissolution of $A$ makes the SR layer locate at the top boundary. The contributions of three chemical species to fluid density are the same, and thus L2 with local minimum density develops below RF and, more specifically, at SD. After the initial diffusion, dense fingers sink from L1 towards the host fluid, and three classical stages of fingering development are observed [Figs. 4(b)-4(e)]. Note that fingering tips can penetrate L2 and become narrow below L2 but wide above [Figs. 4(c)-4(e)]. Actually, L2 can form a buoyantly stable stratification with L3 to hinder fingering progression. In this case, however, the fast diffusion of $B$ can lead to the accumulation of $C$ and thus the increase of fluid density above RF, and the amplitude of the barrier L2 is therefore too weak to counteract the fingering development. 

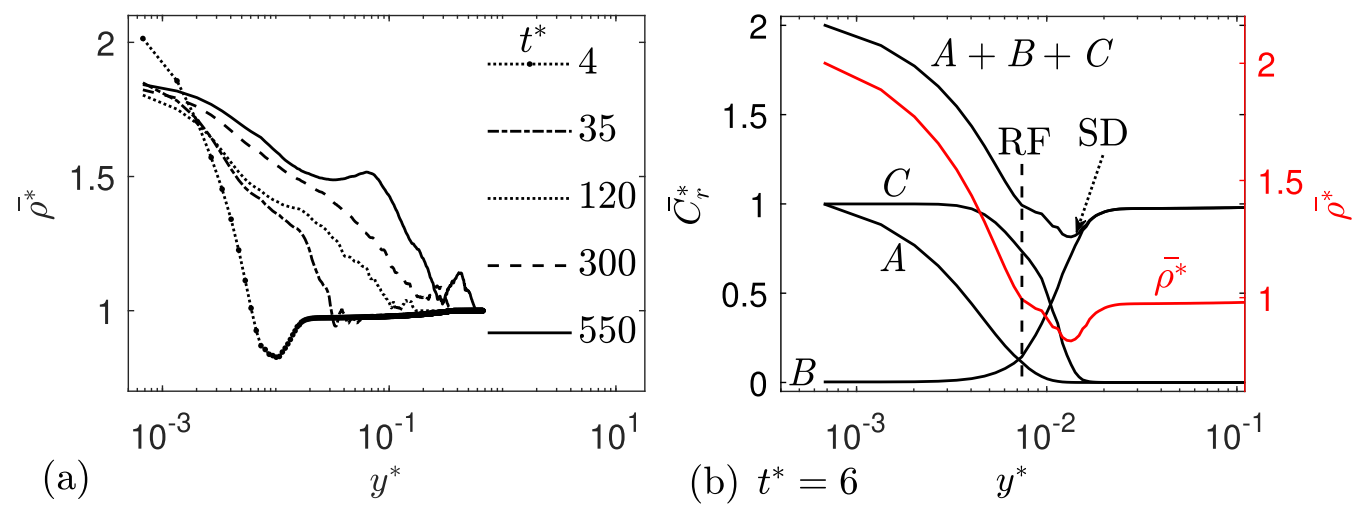

FIG. 5. Horizontally averaged density and species profiles in homogeneous media for case $\mathrm{P} 3$ with $\mathrm{Ra} A=$ $10^{9}, D_{B} / D_{C}=5.0$, and $\mathrm{Ra}_{B} / \mathrm{Ra}_{C}=1.0$ at five different time instants (a) and time instant $t^{*}=6(\mathrm{~b})$.

Concentration distributions in Figs. 4(f)-4(h) corresponding to the density field in Fig. 4(e) also express that a small part of $C$ can penetrate $\mathrm{L} 2$ to form narrow fingers below $\mathrm{L} 2$, and fingers of $A$ play a vital role in wide fingers above. In addition, as seen from Fig. 5(a), the horizontally averaged density curve has a minimum at first but changes into a monotonically decreasing one during the late period. This is caused by the fact that the penetration of fingering tips can disorder the local minimum density layer L2.

CaseP6. After some simulations, density evolutions at $D_{B} / D_{C}=0.1, \mathrm{Ra}_{A}=-10^{9}$, and $\mathrm{Ra}_{B} / \mathrm{Ra}_{C}=1$ are provided in Fig. 6 to illustrate the fingering features in this case. During the initial diffusion, a density stratification is generated as a less dense fluid layer L1 lies above a layer L2 with local maximum density, and L2 locating below RF is followed by a layer L3 [Figs. 6(a)]. Similar to case P2, a DLC mechanism comes into play, resulting in a species distribution with a local SD area at RF and a local SR region below [Fig. 7(b)]. Considering that fluid density decreases with the dissolution of $A$ but increases with the production of $C$, a local maximum layer develops below $\mathrm{RF}$ and specifically at SR, where all of $A$ is consumed by reaction [Fig. 7(b)]. Note that the initial density distribution is different from that in case $\mathrm{P} 2$, and no local minimum density layer appears corresponding to the local SD area. This is because dissolved species $A$ decreases fluid density and introduces a minimum at the top boundary.

Fingering then appears from L2 and experiences three fingering development stages [Figs. 6(b)-6(e)]. It is emphasized that fingering develops slowly in this case and generally becomes weak. This is related to the fact that the density difference between L2 and L3 is too weak to deform the unstable fluid layer quickly, and species $A$ and $B$ tend to diffuse to meet and react with each other. This can be verified by the relative flat interfaces in concentration fields of $A$ and $B$ [Figs. 6(f)-6(g)]. With the chemical consumption of $B$ and the slight deformation of the interface between $A$ and $B$, the concentration of $B$ in the host fluid is diluted generally, and its concentration gradient also decreases. As a consequence, the upward diffusion of $B$ and the production of $C$ by reaction are slowed down. Seen from concentration fields in Figs. 6(f)-6(h), fingers of $C$ are responsible for density fingering, and thus less $C$ results in weaker DI. In addition, it is important to stress the characteristic base shape not observed in other cases with $\mathrm{Ra}_{A}>0$ : a stagnant liquid layer (SLL) near the top boundary [Fig. 6(d)]. As displayed in Fig. 6(f), the remaining $A$ after reaction in the top layer decreases fluid density and contributes to this specific fingering base. Besides, SLL is observed to be pretty thick and spans the entire width of the domain. This also verifies the above explanation that reaction between $A$ and $B$ mainly depends on diffusion, and dissolving $A$ cannot be consumed efficiently and tends to accumulate near the top boundary. In terms of the horizontally averaged density profiles in Fig. 7(a), they illustrate a nonmonotonic fashion with a local maximum, but the amplitude of the maximum decreases with time owing to the dilution of species $B$ in the host fluid. 


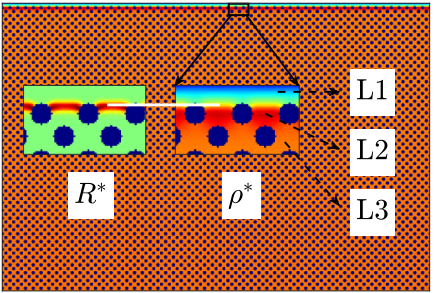

(a) $t^{*}=13$

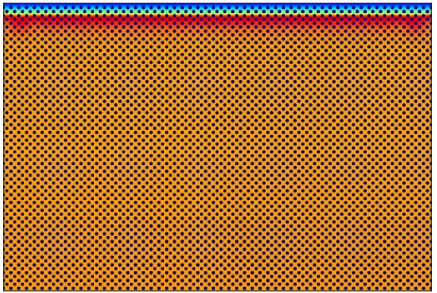

(b) $t^{*}=300$

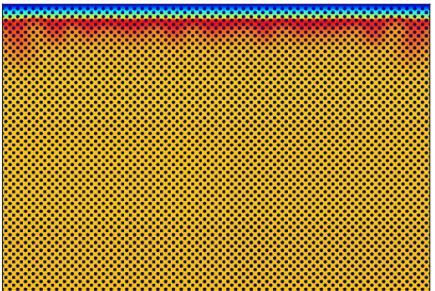

(c) $t^{*}=600$

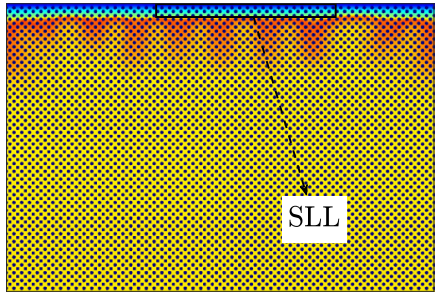

(d) $t^{*}=900$

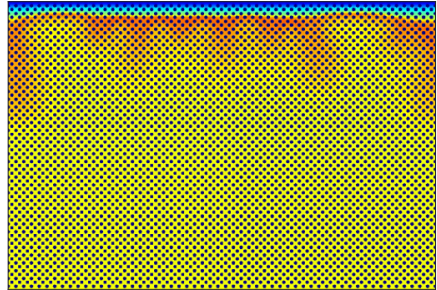

(e) $t^{*}=1200$
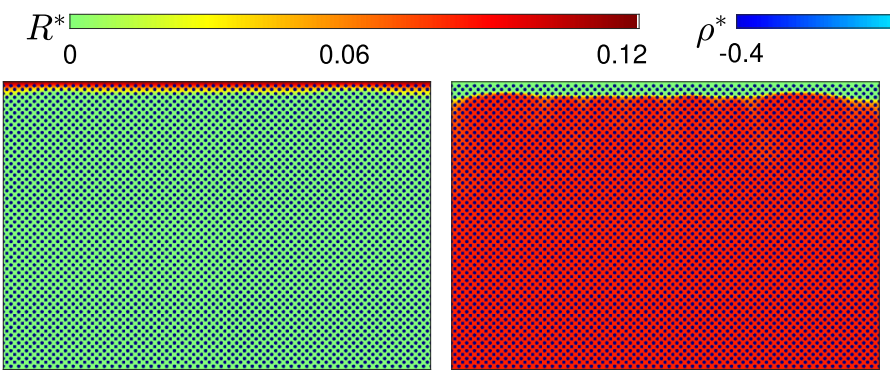

1.5
(f) $t^{*}=1200 \quad C_{A}^{*}$
(g) $t^{*}=1200 \quad C_{B}^{*}$

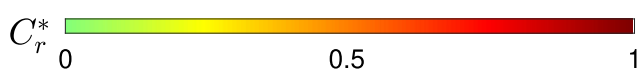

(h) $t^{*}=1200 \quad C_{C}^{*}$

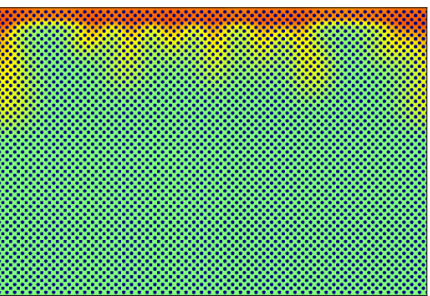

1

FIG. 6. Density (a)-(e) and concentration (f)-(h) distributions in homogeneous media for case P6 with $\mathrm{Ra}_{A}=-10^{9}, D_{B} / D_{C}=0.1$, and $\mathrm{Ra}_{B} / \operatorname{Ra}_{C}=1.0$ at different time instants $t^{*}$. The density scale $\rho^{*}$ varies within the range $-0.4-1.5$; the concentrations $C_{r}^{*}$ scale between 0 and 1 ; and the reaction rate $R^{*}$ scales between 0 and 0.12 .
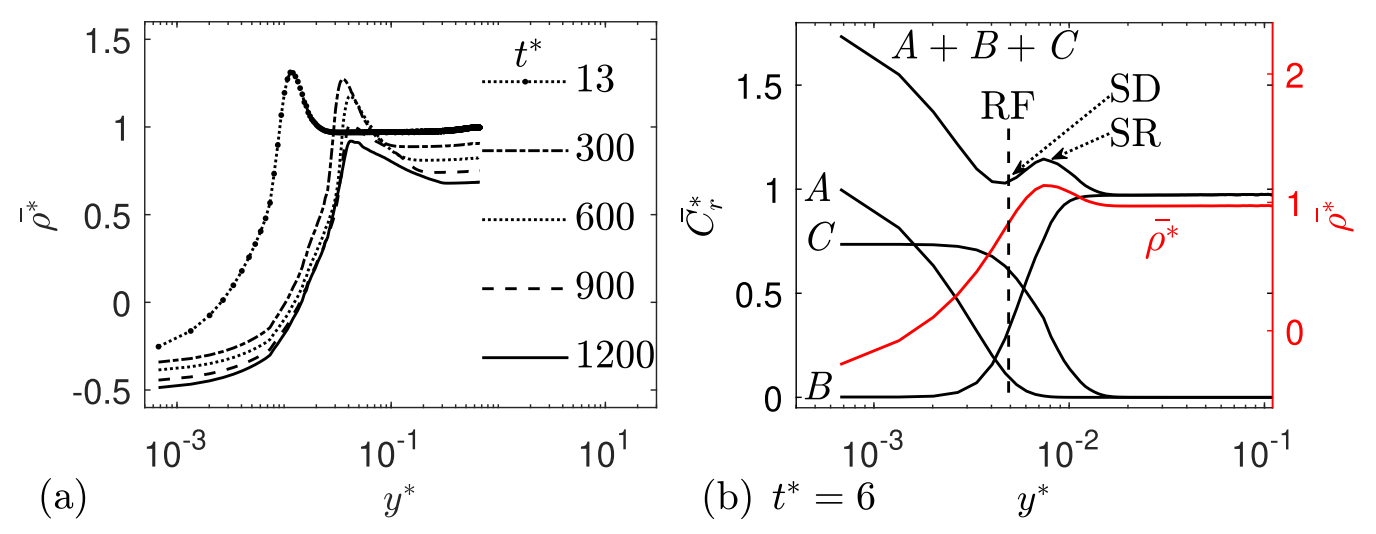

FIG. 7. Horizontally averaged density and species profiles in homogeneous media for case $\mathrm{P} 6$ with $\mathrm{Ra}_{A}=$ $-10^{9}, D_{B} / D_{C}=0.1$, and $\mathrm{Ra}_{B} / \mathrm{Ra}_{C}=1.0$ at five different time instants (a) and time instant $t^{*}=6(\mathrm{~b})$. 


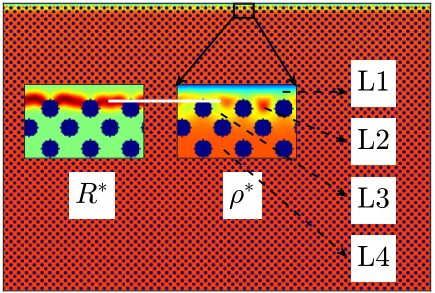

(a) $t^{*}=24$

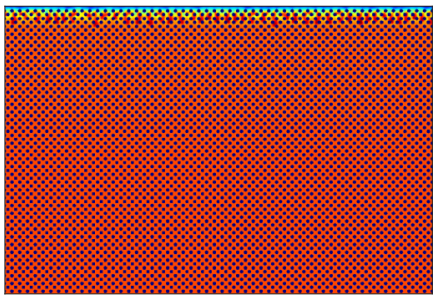

(b) $t^{*}=60$

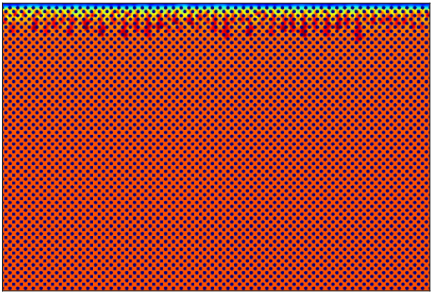

(c) $t^{*}=120$

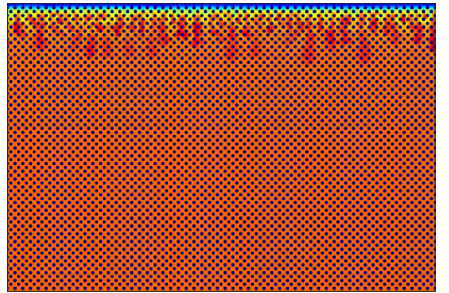

(d) $t^{*}=240$

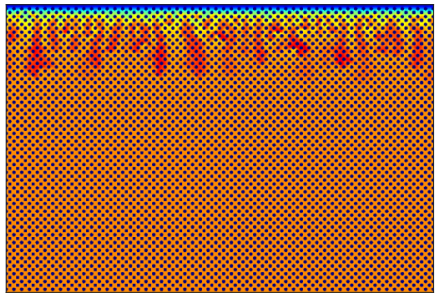

(e) $t^{*}=400$

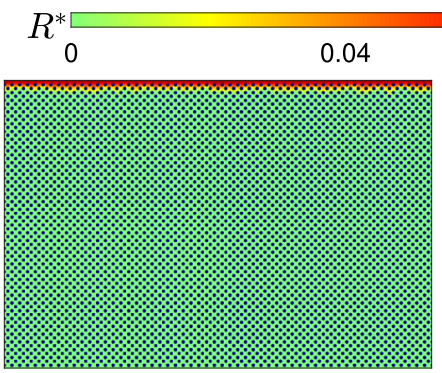

(f) $t^{*}=400 \quad C_{A}^{*}$

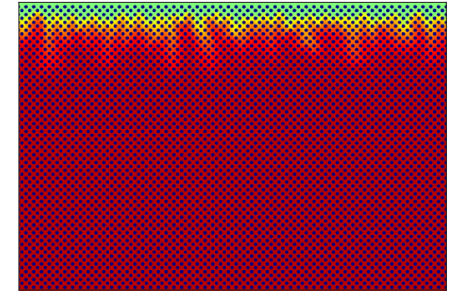

(g) $t^{*}=400 \quad C_{B}^{*}$
0.5 1.3

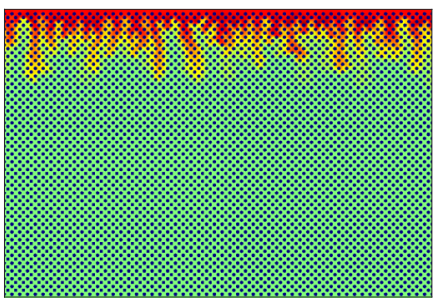

(h) $t^{*}=400 \quad C_{C}^{*}$

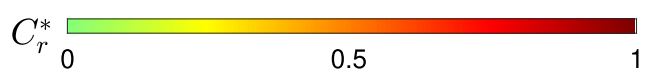

FIG. 8. Density (a)-(e) and concentration (f)-(h) distributions in homogeneous media for case P7 with $\mathrm{Ra}_{A}=-10^{9}, D_{B} / D_{C}=5.0$, and $\mathrm{Ra}_{B} / \mathrm{Ra}_{C}=1.0$ at different time instants $t^{*}$. The density scale $\rho^{*}$ varies within the range -0.3 to 1.3 ; the concentrations $C_{r}^{*}$ scale between 0 and 1 ; and the reaction rate $R^{*}$ scales between 0 and 0.08 .

It should be pointed out that fingering is not observed in the equal diffusivity counterpart, and the local maximum density L2 (or DLC mechanism) is at the origin of density fingering.

CaseP7. Fingering phenomena in this case are finally simulated, and results at $D_{B} / D_{C}=5$, $\mathrm{Ra}_{A}=-10^{9}$, and $\mathrm{Ra}_{B} / \mathrm{Ra}_{C}=1.0$ are displayed in Fig. 8 for introduction. Initial diffusion can introduce a density stratification of four layers: a less dense layer L1 lies on layer L2 with local maximum density, and the following layer L3 with local minimum density overlies a denser one L4 [Fig. 8(a)]. This stratification is generated due to the DD-induced species distribution with a local SD area below RF [Fig. 9(b)]. Specifically, under the condition that fluid density decreases with dissolved $A$ but increases with product $C$, a local maximum density layer L2 forms at RF where less $A$ but more $C$ is present, and a local minimum layer L3 is built up at the local SD region where no species $C$ exists [Fig. 9(b)]. In this case, only one unstable stratification is formed by L2 and L3, and DI develops from L2 after the initial diffusion [Figs. 8(b)-8(e)]. Even DD-caused L3 can form a buoyantly stable stratification with L4, and fingering tips can penetrate this weak barrier, owing to the accumulation of species $C$ above RF. After this penetration, fingering becomes strong below L3, because species $A$ decreases fluid density obviously in the top layer. It is emphasized 

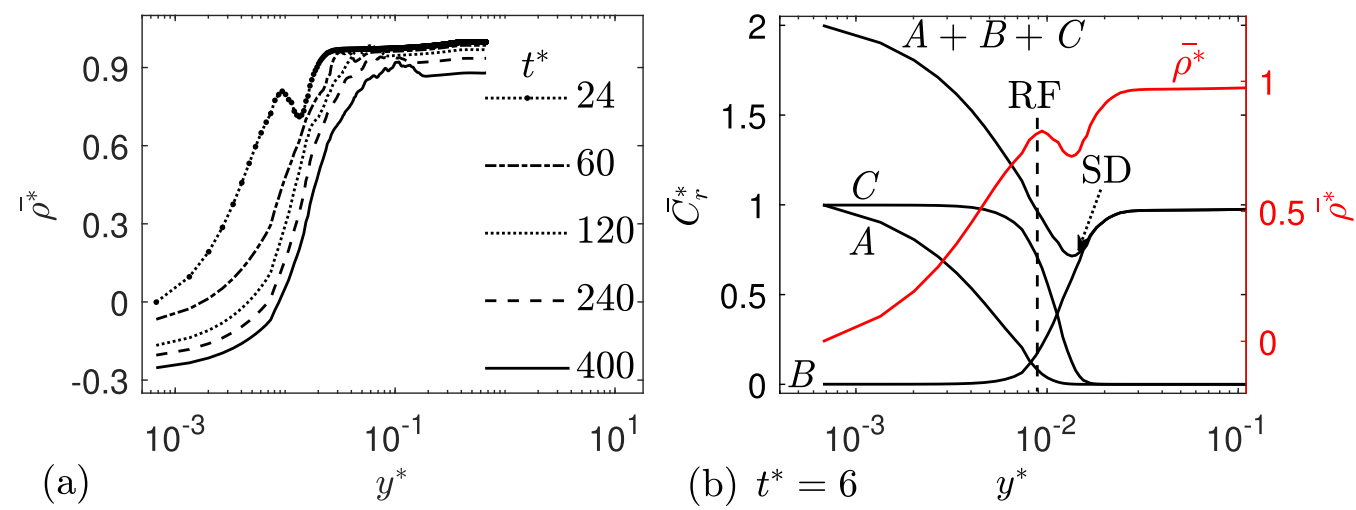

FIG. 9. Horizontally averaged density and species profiles in homogeneous media for case $\mathrm{P} 7$ with $\mathrm{Ra}_{A}=$ $-10^{9}, D_{B} / D_{C}=5.0$, and $\mathrm{Ra}_{B} / \mathrm{Ra}_{C}=1.0$ at five different time instants (a) and time instant $t^{*}=6$ (b).

that, similar to case P6, fingering grows slowly with the decreases of solute $B$ in the host fluid, and SLL caused by remaining $A$ near the top boundary is also observed. As for the calculated density curves in Fig. 8(1f), they initially show a nonmonotonic pattern with a maximum followed by a minimum, which is similar to the density field in Fig. 8(a). But generally, the density line changes into a nonmonotonic fashion with a local maximum, and the value of local maximum decreases with time. The disappearance of a local minimum can be explained by the penetration of fingering tips as in case P3, and the decrease of a local maximum is due to the dilution of $B$ as in case P6. Considering that no fingering develops in the equal diffusivity counterpart, the DD-induced unstable stratification of L2 and L3 (also DD mechanism) can introduce the development of DI.

To summarize, the development of DI can be delineated into eight cases depending on parameters $D_{r}$ and $\mathrm{Ra}_{r}$. Fingering properties of cases P1, P4, P5, and P8 are consistent with those of cases R1-R4 in our recent equal diffusivity simulations [27], and they are mainly determined by chemical reaction. In the other four cases, however, the effects of differential diffusion become obvious, and DD and DLC mechanisms can introduce new fingering features not observed in Ref. [27]. Specifically, a DLC-induced maximum can bring in the second DI in P2; a DD-induced minimum can be penetrated by fingering tips in P3; and DD and DLC mechanisms can even trigger the development of DI in cases P6 and P7. The present classification is generally consistent with the theoretical one in Ref. [23], and calculated horizontally averaged density profiles agree well with theoretical predictions in the early period: monotonically increasing (P1) or decreasing (P8), with one minimum below (P3) or at (P4) RF, with one maximum at (P5) or below (P6) RF, with a minimum followed by maximum (P2) or the opposite (P7). As fingering reaches the late nonlinear stage, however, averaged density curves tend to increase monotonically (P2, P3) or increase nonmonotonically with a local maximum (P6, P7). Besides, another difference between the present result and the theoretical analysis in Ref. [23] is that species distributions can be obtained in the present numerical simulations, and local SD and SR areas can be clearly captured to identify the positions of local minimum and maximum density layers.

\section{B. Quantitative effects}

Having investigated the general phenomena of DI, a quantitative study is further carried out. To focus on the impact of differential diffusion, all species are assumed to contribute equally to fluid density, namely, $\operatorname{Ra}_{A}=\mathrm{Ra}_{B}=\mathrm{Ra}_{C}=10^{9}$. Besides, the diffusion rate of $B$ is fixed as $D_{B}=D_{A}$, and different values of $D_{C}$ are selected to vary $D_{B} / D_{C}$ from 0.1 to 10 .

Effects of differential diffusion on the progression of DI are first evaluated by calculating the mixing length $l_{m}$. This quantity $l_{m}$ is defined as the most advanced vertical position of fingering 


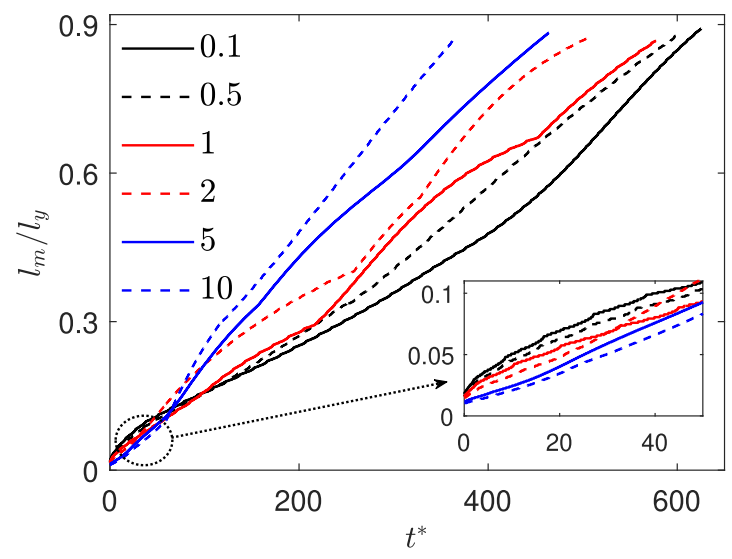

FIG. 10. Time evolutions of the mixing length in homogeneous media for tests with $\operatorname{Ra}_{A}=\operatorname{Ra}_{B}=\operatorname{Ra}_{C}=$ $10^{9}$ and $D_{B} / D_{C}=0.1,0.5,1,2,5,10$.

tips in the host fluid. Temporal evolutions of $l_{m}$ in Fig. 10 indicate that $l_{m}$ increases with decreasing $D_{B} / D_{C}$ during the early diffusion stage. This is because the relative diffusion of $C$ becomes larger with smaller $D_{B} / D_{C}$, and the top fluid layer rich in species $A$ and $C$ can progress into the host fluid faster, leading to larger $l_{m}$. After this short period, $l_{m}$ changes to increase with time rapidly due to the appearance of fingering. It is found that in tests with larger $D_{B} / D_{C}, l_{m}$ deviates from the initial diffusive trend faster and grows to reach the bottom earlier. This is because the larger $D_{B} / D_{C}$ indicates the fast-diffusing $B$ and the slow-diffusing $C$. First, slowly diffusing $C$ tends to accumulate in the top layer and increase fluid density. Second, fast-diffusing $B$ can lead to intensive reaction and more product $C$. The combination of these two factors contributes to intensifying the unstable stratification and then promoting the appearance and the progression of DI.

Considering that the dissolution of species $A$ into the host fluid is always desirable in related applications, effects of differential diffusion on the storage efficiency of $A$ are also quantitatively investigated. As conducted in Ref. [27], two metrics are calculated. The first one is the horizontally averaged mass flux of species $A$ at the top boundary [27],

$$
J^{*}(t)=-\frac{1}{l_{x}^{*} \sqrt{\mathrm{Ra}_{A} \mathrm{Sc}}} \int_{0}^{l_{x}^{*}} \partial_{y^{*}} C_{A}^{*}\left(x^{*}, 0\right) d x^{*} .
$$

This parameter can be regarded as an indicator for the diffusion speed of $A$ into the host fluid. The second one is the amount of $A$ stored in the host fluid, which is defined as the volume-averaged concentration $\left\langle C_{A}^{*}+C_{C}^{*}\right\rangle$ [4]. As illustrated in Fig. 11, each $J^{*}$ evolves with time nonmonotonically at first and finally fluctuates around a steady-state value, and each $\left\langle C_{A}^{*}+C_{C}^{*}\right\rangle$ increases with time monotonically.

Regardless of the similar development pattern, deficiencies introduced by differential diffusion are obvious in Fig. 11. Every $J^{*}$ with differential diffusion is smaller than the equal diffusivity one, and the difference amplifies when the value of $D_{B} / D_{C}$ departs from $D_{B} / D_{C}=1$ more obviously [Fig. 11(a)]. This can be explained as follows. First, for tests with $D_{B} / D_{C}<1$, a local minimum density layer develops at RF due to the DLC mechanism as in case P2 (Fig. 2), and this minimum can suppress the development of DI as well as the accompanied convection. Thus, less $B$ can be brought up to react with $A$, and the chemical consumption of $A$ slows down, leading to small $J^{*}$. With the increase of $D_{B} / D_{C}$, the intensity of DLC and the inhibition effects of local minimum become weak, and subsequently, the value of $J^{*}$ increases. The influence of DLC finally disappears when $D_{B} / D_{C}$ increases to 1 and $J^{*}$ increases to reach its maximum value. As $D_{B} / D_{C}$ continues growing to become larger than 1, the DD mechanism comes into play and introduces a local minimum layer below RF (case P3 in Fig. 4). Similarly, this minimum layer can suppress the dissolution of $A$, 

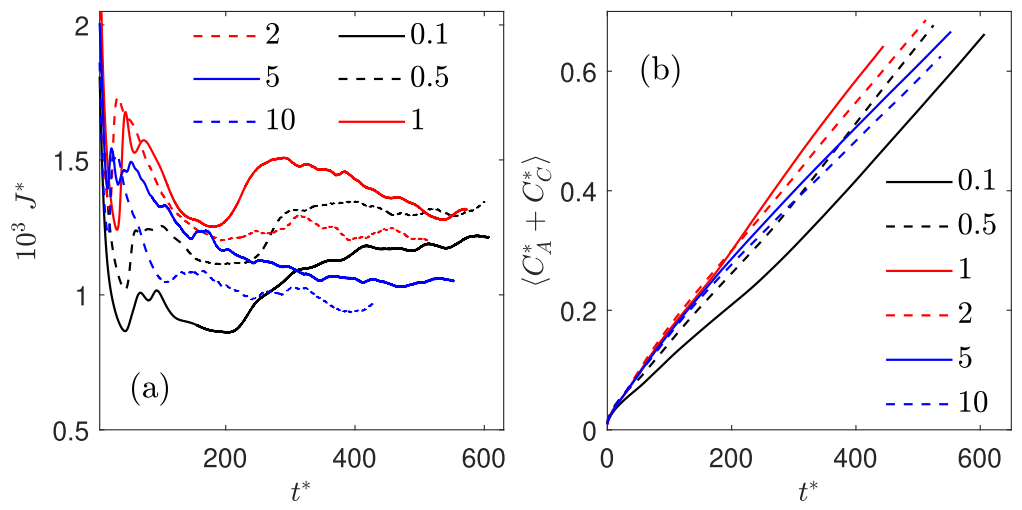

FIG. 11. Time evolutions of the storage of species $A$ in homogeneous media for tests with $\operatorname{Ra}_{A}=\mathrm{Ra}_{B}=$ $\operatorname{Ra}_{C}=10^{9}$ and $D_{B} / D_{C}=0.1,0.5,1,2,5,10$.

and its intensity increases with increasing $D_{B} / D_{C}$. That is, with increasing $D_{B} / D_{C}, J^{*}$ increases if $D_{B} / D_{C}<1$ but decreases if $D_{B} / D_{C}>1$. Similar to results of $J^{*}$, the obtained lines for $\left\langle C_{A}^{*}+C_{C}^{*}\right\rangle$ in Fig. 11(b) also show that the largest amount of $A$ can be dissolved into the host fluid if $D_{B} / D_{C}=1$, and the stored amount decreases with increasing $D_{B} / D_{C}$ if $D_{B} / D_{C}>1$ or with decreasing $D_{B} / D_{C}$ if $D_{B} / D_{C}<1$. Thus, calculated $J^{*}$ and $\left\langle C_{A}^{*}+C_{C}^{*}\right\rangle$ suggest that differential diffusion can suppress the storage of species $A$ in the host fluid, and the inhibition extent increases with the increasing difference between the diffusion rates of $B$ and $C$. In addition, this conclusion has been checked for other values of $\mathrm{Ra}_{B} / \mathrm{Ra}_{C}$.

\section{CONCLUSIONS}

Pore-scale simulations of density-driven instability (DI) with reaction $A+B \rightarrow C$ and differential diffusion have been conducted in a porous medium with a partially miscible top boundary using an MRT LB model. Numerical results demonstrate eight types of DI depending on $\mathrm{Ra}_{A}$, $\mathrm{Ra}_{B} / \mathrm{Ra}_{C}$, and $D_{B} / D_{C}$, which generally confirm previous theoretical classifications. Compared with our recent equal diffusivity simulations in Ref. [27], the inclusion of differential diffusion brings in the double-diffusive (DD) and the diffusive-layer convection (DLC) mechanisms and subsequently gives rise to four new DI dynamics. On the one hand, when $D_{B} / D_{C}>1$ and $\mathrm{Ra}_{B} / \mathrm{Ra}_{C}$ is small, the DD mechanism can produce a local species-depleted (SD) area below the reaction front (RF) and then brings in a local minimum density layer at SD. In case P3 with $\mathrm{Ra}_{A}>0$, this minimum can suppress fingering development, even it can be penetrated by finger tips finally. Note that fingering becomes narrow below the minimum but large above. On the other hand, if $D_{B} / D_{C}<1$ and $\mathrm{Ra}_{B} / \mathrm{Ra}_{C}$ is large, the DLC can introduce a local SD area at RF followed by a local species-rich (SR) region and subsequently brings in a local density maximum layer at SR. This DLC-caused maximum thus leads to the second DI and the antenna-shaped fingers in case P2 with $\mathrm{Ra}_{A}>0$. In the meantime, for cases P5 and P6 with $\mathrm{Ra}_{A}<0$, the corresponding equal diffusivity cases are stable, but the DD-induced minimum and DLC-caused maximum can trigger the development of DI. In these four cases with obvious differential diffusion effects, density curves are initially similar to theoretical results but generally tend to decrease monotonically (P2 and P3) or increase nonmonotonically with a local maximum (P6 and P7). In addition, different from previous theoretical studies, local SD and SR regions can be captured in the present simulations to better define the locations of local minimum or maximum density layers.

Finally, the role of differential diffusion has been highlighted by a series of parametric simulations and quantitative comparisons. It is concluded that with increasing $D_{B} / D_{C}$, fingering tips progress into the host fluid more slowly during the initial diffusion stage but finally tend to grow more rapidly and reach the bottom much earlier. In terms of the storage of species $A$ in the host fluid, 
it changes nonlinearly with increasing $D_{B} / D_{C}$ : the largest amount of $A$ can be stored in the host fluid when $D_{B}=D_{C}$, and the storage efficiency decreases with increasing difference between $D_{B}$ and $D_{C}$. Thus differential diffusion tends to suppress the dissolution of $A$ from the top boundary. The present pore-scale results suggest that understanding the effects of differential diffusion is of great importance for related applications. Specifically, in the context of $\mathrm{CO}_{2}$ sequestration, comparing chemical compositions in different geological storage sites, and selecting those where reaction and differential diffusion can enhance the dissolution of $\mathrm{CO}_{2}$ is crucial to improving the storage efficiency and safety.

\section{ACKNOWLEDGMENTS}

This work was supported by the UK Engineering and Physical Sciences Research Council, under the UK Consortium on Mesoscale Engineering Sciences (UKCOMES) (Grants No. EP/R029598/1), and the High-Level Mesoscale Modelling System (HiLeMMS) (Grant No. EP/P022243/1), and the China Scholarship Council (CSC) under Grant No. 201706160161.

\section{APPENDIX: THE MRT LB MODEL}

In this Appendix, the two-dimensional nine-velocity MRT LB model for the above pore-scale simulations is introduced. It should be emphasized that this model is generally similar to the one developed in our recent work [27], except for the different diffusion rates $D_{r}$ of species $A, B$, and $C$. Specifically, the LB equations for the NS equations (3) and (4) and the CDR equations (5)-(7) are introduced as $[24,27,30]$

$f_{i}\left(\boldsymbol{x}+\boldsymbol{e}_{i} \delta_{t}, t+\delta_{t}\right)-f_{i}(\boldsymbol{x}, t)=-\left(\boldsymbol{M}^{-1} \boldsymbol{S} \boldsymbol{M}\right)_{i j}\left[f_{j}(\boldsymbol{x}, t)-f_{j}^{e q}(\boldsymbol{x}, t)\right]+\delta_{t}\left[\boldsymbol{M}^{-1}\left(\boldsymbol{I}-\frac{\boldsymbol{S}}{2}\right) \boldsymbol{M}\right]_{i j} \bar{F}_{j}$,

$g_{i, r}\left(\boldsymbol{x}+\boldsymbol{e}_{i} \delta_{t}, t+\delta_{t}\right)-g_{i, r}(\boldsymbol{x}, t)=-\left(\boldsymbol{M}^{-1} \boldsymbol{S}_{c, r} \boldsymbol{M}\right)_{i j}\left[g_{j, r}(\boldsymbol{x}, t)-g_{j, r}^{e q}(\boldsymbol{x}, t)\right]+\delta_{t} \bar{R}_{i, r}+\frac{\delta_{t}^{2}}{2} \partial_{t} \bar{R}_{i, r}$,

for $i, j=0,1, \ldots, 8$, where $f_{i}(\boldsymbol{x}, t)$ and $g_{i, r}(\boldsymbol{x}, t)$ are the distribution functions for the hydrodynamics and the concentration field of species $r$, respectively. The time derivative term is treated by the backward scheme as $\partial_{t} \bar{R}_{i, r}(\boldsymbol{x}, t)=\left[\bar{R}_{i, r}(\boldsymbol{x}, t)-\bar{R}_{i, r}\left(\boldsymbol{x}, t-\delta_{t}\right)\right]$. The transformation matrix $\boldsymbol{M}$ can map the distribution functions from the physical space to the moment space. $\boldsymbol{S}$ and $\boldsymbol{S}_{c, r}$ are the diagonal relaxation matrix of relaxation rates $s_{i}$ and $s_{i c, r}$ in the moment space, respectively. In the following simulations, the relaxation rates are chosen as those in Refs. [30,32], and the corresponding discrete velocities $\boldsymbol{e}_{i}$ and weight coefficients $w_{i}$ are set as in Ref. [24].

To recover the incompressible NS equations correctly, the equilibrium distribution functions $f_{i}^{e q}$ and $g_{i, r}^{e q}$ are given as [24,27]

$$
\begin{gathered}
f_{i}^{e q}=w_{i}\left[\rho_{p}+\rho_{0}\left(\frac{\boldsymbol{e}_{i} \cdot \boldsymbol{u}}{c_{s}^{2}}+\frac{\boldsymbol{u} \boldsymbol{u}:\left(\boldsymbol{e}_{i} \boldsymbol{e}_{i}-c_{s}^{2} \boldsymbol{I}\right)}{2 c_{s}^{4}}\right)\right], \\
g_{i, r}^{e q}=w_{i} C_{r}\left[1+\frac{\boldsymbol{e}_{i} \cdot \boldsymbol{u}}{c_{s}^{2}}+\frac{\boldsymbol{u} \boldsymbol{u}:\left(\boldsymbol{e}_{i} \boldsymbol{e}_{i}-c_{s}^{2} \boldsymbol{I}\right)}{2 c_{s}^{4}}\right] .
\end{gathered}
$$

Here $\rho_{p}$ is a variable related to the fluid pressure as $p=c_{s}^{2} \rho_{p}$, with $c_{s}=e / \sqrt{3}$ being the lattice sound velocity. To avoid discrete lattice effects in the LB model, the forcing and reactive distribution 
functions $\bar{F}_{i}$ and $\bar{R}_{i, r}$ are [24,27]

$$
\begin{gathered}
\bar{F}_{i}=w_{i}\left[\frac{\boldsymbol{e}_{i} \cdot \boldsymbol{F}}{c_{s}^{2}}+\frac{\boldsymbol{u} \boldsymbol{F}:\left(\boldsymbol{e}_{i} \boldsymbol{e}_{i}-c_{s}^{2} \boldsymbol{I}\right)}{c_{s}^{4}}\right], \\
\bar{R}_{i, r}=w_{i} R_{r}\left(1+\frac{\boldsymbol{e}_{i} \cdot \boldsymbol{u}}{c_{s}^{2}} \frac{\tau_{c, r}-0.5}{\tau_{c, r}}\right),
\end{gathered}
$$

with $R_{r}$ being the reaction rate in the CDR equation for species $r$.

Finally, the macroscopic variables can be obtained from the distribution functions as

$$
\rho_{p}=\sum_{i} f_{i}, \quad \rho_{0} \boldsymbol{u}=\sum_{i} \boldsymbol{e}_{i} f_{i}+\frac{\delta_{t}}{2} \boldsymbol{F}, \quad C_{r}=\sum_{i} g_{r} .
$$

Through the Chapman-Enskog analysis on the present LB equations, the governing equations (3)(7) can be recovered with the relaxation times $\tau$ and $\tau_{c, r}$ as follows:

$$
v=c_{s}^{2}\left(\tau-\frac{1}{2}\right) \delta_{t}, \quad D_{r}=c_{s}^{2}\left(\tau_{c, r}-\frac{1}{2}\right) \delta_{t} .
$$

In addition, the characteristic parameters in Eq. (11) are set as

$$
L=l_{x}=1, \quad U=\sqrt{g \beta_{A} L C_{A 0}}=0.177, \quad T=L / U=5.65, \quad C_{d}=C_{A 0}=1,
$$

and they are all in lattice units. Based on these characteristic numbers, the relevant parameters in LB simulations can be calculated as

$$
\begin{aligned}
v & =\frac{L U}{\sqrt{\mathrm{Ra}_{A} / \mathrm{Sc}}}, \quad D_{A}=\frac{L U}{\sqrt{\mathrm{Ra}_{A} \mathrm{Sc}}}, \quad D_{B}=D_{B}^{*} D_{A}, \quad D_{C}=D_{C}^{*} D_{A}, \\
C_{B 0} & =C_{A 0} \eta, \quad \tau=\frac{v}{c_{s}^{2} \delta_{t}}+\frac{1}{2}, \quad \tau_{c, r}=\frac{D_{r}}{c_{s}^{2} \delta_{t}}+\frac{1}{2}, \quad k=\frac{U \mathrm{Da}}{L C_{A 0}} .
\end{aligned}
$$

[1] D. Loggia, D. Salin, and Y. C. Yortsos, The effect of dispersion on the stability of non-monotonic mobility profiles in porous media, Phys. Fluids 10, 747 (1998).

[2] O. Manickam and G. M. Homsy, Fingering instabilities in vertical miscible displacement flows in porous media, J. Fluid Mech. 288, 75 (1995).

[3] C. Thomas, S. Dehaeck, and A. De Wit, Convective dissolution of $\mathrm{CO}_{2}$ in water and salt solutions, Int. J. Greenhouse Gas Control 72, 105 (2018).

[4] V. Loodts, B. Knaepen, L. Rongy, and A. De Wit, Enhanced steady-state dissolution flux in reactive convective dissolution, Phys. Chem. Chem. Phys. 19, 18565 (2017).

[5] R. A. Wooding, S. W. Tyler, and I. White, Convection in groundwater below an evaporating Salt Lake: 1. Onset of instability, Water Resour. Res. 33, 1199 (1997).

[6] J. D. Stevens, J. M. Sharp, C. T. Simmons, and T. R. Fenstemaker, Evidence of free convection in groundwater: Field-based measurements beneath wind-tidal flats, J. Hydrol. 375, 394 (2009).

[7] L. Rongy, K. B. Haugen, and A. Firoozabadi, Mixing from Fickian diffusion and natural convection in binary non-equilibrium fluid phases, AIChE J. 58, 1336 (2012).

[8] M. A. Budroni, L. A. Riolfo, L. Lemaigre, F. Rossi, M. Rustici, and A. De Wit, Chemical control of hydrodynamic instabilities in partially miscible two-layer systems, J. Phys. Chem. Lett. 5, 875 (2014).

[9] C. Wylock, A. Rednikov, P. Colinet, and B. Haut, Experimental and numerical analysis of buoyancyinduced instability during $\mathrm{CO}_{2}$ absorption in $\mathrm{NaHCO}_{3}-\mathrm{Na}_{2} \mathrm{CO}_{3}$ aqueous solutions, Chem. Eng. Sci. 157, 232 (2017).

[10] I. Cherezov and S. S. S. Cardoso, Acceleration of convective dissolution by chemical reaction in a HeleShaw cell, Phys. Chem. Chem. Phys. 18, 23727 (2016). 
[11] M. A. Budroni, C. Thomas, and A. De Wit, Chemical control of dissolution-driven convection in partially miscible systems: Nonlinear simulations and experiments, Phys. Chem. Chem. Phys. 19, 7936 (2017).

[12] C. Thomas, L. Lemaigre, A. Zalts, A. D'Onofrio, and A. De Wit, Experimental study of $\mathrm{CO}_{2}$ convective dissolution: The effect of color indicators, Int. J. Greenhouse Gas Control 42, 525 (2015).

[13] M. C. Kim, Effect of the irreversible $A+B \rightarrow C$ reaction on the onset and the growth of the buoyancydriven instability in a porous medium, Chem. Eng. Sci. 112, 56 (2014).

[14] M. C. Kim and C. Wylock, Linear and nonlinear analyses of the effect of chemical reaction on the onset of buoyancy-driven instability in $\mathrm{CO}_{2}$ absorption process in a porous medium or Hele-Shaw cell, Can. J. Chem. Eng. 95, 589 (2017).

[15] V. Loodts, C. Thomas, L. Rongy, and A. De Wit, Control of Convective Dissolution by Chemical Reactions: General Classification and Application to $\mathrm{CO}_{2}$ Dissolution in Reactive Aqueous Solutions, Phys. Rev. Lett. 113, 114501 (2014).

[16] V. Loodts, L. Rongy, and A. De Wit, Chemical control of dissolution-driven convection in partially miscible systems: Theoretical classification, Phys. Chem. Chem. Phys. 17, 29814 (2015).

[17] C. Wylock, A. Rednikov, B. Haut, and P. Colinet, Nonmonotonic Rayleigh-Taylor instabilities driven by gas-liquid $\mathrm{CO}_{2}$ chemisorption, J. Phys. Chem. B 118, 11323 (2014).

[18] C. Thomas, V. Loodts, L. Rongy, and A. De Wit, Convective dissolution of $\mathrm{CO}_{2}$ in reactive alkaline solutions: Active role of spectator ions, Int. J. Greenhouse Gas Control 53, 230 (2016).

[19] V. Loodts, H. Saghou, B. Knaepen, L. Rongy, and A. De Wit, Differential diffusivity effects in reactive convective dissolution, Fluids 3, 83 (2018).

[20] P. M. J. Trevelyan, C. Almarcha, and A. De. Wit, Buoyancy-driven instabilities of miscible two-layer stratifications in porous media and Hele-Shaw cells, J. Fluid Mech. 670, 38 (2011).

[21] J. Carballido-Landeira, P. M. J. Trevelyan, C. Almarcha, and A. De Wit, Mixed-mode instability of a miscible interface due to coupling between Rayleigh-Taylor and double-diffusive convective modes, Phys. Fluids 25, 024107 (2013).

[22] P. M. J. Trevelyan, C. Almarcha, and A. De Wit, Buoyancy-driven instabilities around miscible $A+B \rightarrow$ C reaction fronts: A general classification, Phys. Rev. E 91, 023001 (2015).

[23] V. Loodts, P. M. J. Trevelyan, L. Rongy, and A. De Wit, Density profiles around $A+B \rightarrow C$ reactiondiffusion fronts in partially miscible systems: A general classification, Phys. Rev. E 94, 043115 (2016).

[24] Z. L. Guo and C. Shu, Lattice Boltzmann Method and Its Applications in Engineering (World Scientific Publishers, Singapore, 2013), pp. 25-28, 76-78, 329-338.

[25] Q. Li, K. H. Luo, Q. J. Kang, Y. L. He, Q. Chen, and Q. Liu, Lattice Boltzmann methods for multiphase flow and phase-change heat transfer, Prog. Energy Combust. Sci. 52, 62 (2016).

[26] T. M. Lei, X. H. Meng, and Z. L. Guo, Pore-scale study on reactive mixing of miscible solutions with viscous fingering in porous media, Comput. Fluids 155, 146 (2017).

[27] T. M. Lei and K. H. Luo, Pore-scale study of dissolution-driven density instability with reaction $A+B \rightarrow$ $C$ in porous media, Phys. Rev. Fluids 4, 063907 (2019).

[28] C. Chen and D. Zhang, Pore-scale simulation of density-driven convection in fractured porous media during geological $\mathrm{CO}_{2}$ sequestration, Water Resour. Res. 46, W11527 (2010).

[29] Z. L. Guo and C. G. Zheng, Analysis of lattice Boltzmann equation for microscale gas flows: Relaxation times, boundary conditions and the Knudsen layer, Int. J. Comput. Fluid Dynamics 22, 465 (2008).

[30] X. H. Meng and Z. L. Guo, Multiple-relaxation-time lattice Boltzmann model for incompressible miscible flow with large viscosity ratio and high Péclet number, Phys. Rev. E 92, 043305 (2015).

[31] D. d'Humieres, I. Ginzburg, M. Krafczyk, P. Lallemand, and L. S. Luo, Multiple-relaxation-time lattice Boltzmann models in three dimensions, Philos. Trans. R. Soc. A 360, 437 (2002).

[32] C. X. Pan, L. S. Luo, and C. T. Miller, An evaluation of lattice Boltzmann schemes for porous medium flow simulation, Comput. Fluids 35, 898 (2006).

[33] A. J. C. Ladd, Numerical simulations of particulate suspensions via a discretized Boltzmann equation, Part 1. Theoretical foundation, J. Fluid Mech. 271, 285 (1994).

[34] A. J. C. Ladd, Numerical simulations of particulate suspensions via a discretized Boltzmann equation, Part 2. Numerical results, J. Fluid Mech. 271, 311 (1994). 